\title{
Endovascular Resection of the Prostate: How Much Is Enough for Prostate Artery Embolization?
}

\author{
Tiago Bilhim, MD, PhD, EBIR, FCIRSE, FSIR
}

\section{ABBREVIATIONS}

$\mathrm{BPH}=$ benign prostatic hyperplasia, $\mathrm{CGD}=$ central gland tissue detachment, $\mathrm{PAE}=$ prostate artery embolization, $\mathrm{RCT}=$ randomized controlled trial, TURP $=$ transurethral resection of the prostate

The study by Hechelhammer et al (1) titled "Predictability and Inducibility of Detachment of Prostatic Central Gland Tissue after Prostatic Artery Embolization: Post Hoc Analysis of a Randomized Controlled Trial" is a secondary analysis of data from a recently published randomized controlled trial (RCT) of prostate artery embolization (PAE) versus transurethral resection of the prostate (TURP) for benign prostatic hyperplasia (BPH) (2). The authors studied the incidence of prostatic central gland tissue detachment (CGD) on a prospective cohort of 48 patients who underwent PAE for BPH and looked at predictors of this phenomena. In this cohort of patients who underwent PAE, CGD was present in 3 patients $(6.3 \%)$, with 1 reporting spontaneous elimination of the necrotic prostatic tissue through the urethra. The 2 remaining patients had to undergo endoscopic procedures to remove the necrotic prostatic tissue due to urinary tract obstruction in one and urinary tract infection in the other. The authors identified the following predictors of CGD: patients under acute urinary retention with larger prostate sizes with higher central gland indexes, receiving higher amounts of embolic material. These patients experienced more pain and had higher inflammatory responses during the immediate post-PAE course and during the following week. Ejaculation was assessed in two-thirds of patients with CGD, and complete anejaculation after PAE was reported in both patients. The small sample size limited statistically significant differences, but functional outcomes in the patients with CGD seemed to

From the Centro Hepato-Bílio-Pancreático e da Transplantação, Hospital Curry Cabral, CHULC, Saint Louis Hospital, Rua Luz Soriano, 182, Lisbon, Portugal 1200-249. Received October 9, 2018; accepted October 10, 2018. Address correspondence to T.B.; E-mail: tiagobilhim@hotmail.com

T.B. is a paid consultant for Terumo (Tokyo, Japan) and Merit Medical (South Jordan, Utah), an advisory board member for Merit Medical, a paid speaker for Philips (Eindhoven, The Netherlands), and a shareholder in Embolx (Sunnyvale, California)

(c) $\mathrm{SIR}, 2018$

J Vasc Interv Radiol 2019; 30:225-227

https://doi.org/10.1016/j.jvir.2018.10.009 be more pronounced, with higher increases in peak urinary flowrate $\left(\mathrm{Q}_{\max }\right)$, more pronounced prostate volume, and postvoid residual urine volume (PVR) reductions. International Prostate Symptom Score and quality of life after PAE seemed to be similar between patients with CGD and those without CGD. When looking at the magnetic resonance images of patients with CGD and comparing them with patients without CGD, one is left with the impression that these patients almost have a "post-TURP" appearance with a wideopen bladder neck and a cavity where previously the central gland of the prostate was. These findings of CGD that was subsequently managed endoscopically mimic an assisted "endovascular resection of the prostate." The questions that immediately arise are: Is this a good thing? How much prostate do we want to destroy with PAE? What to do with the necrotic prostatic tissue that is left behind? How to avoid CGD?

CGD with spontaneous elimination of the necrotic prostatic tissue through the urethra has been previously reported in 3 patients $(3,4)$, whereas CGD requiring endoscopic retrieval of the necrotic tissue has been previously reported in another 2 patients $(4,5)$. What do these 8 patients have in common? They were all embolized with spherical embolic agents smaller than $300 \mu \mathrm{m}$ (either 250- $\mu \mathrm{m}$ Embozene microspheres, Boston Scientific, Marlborough Massachusetts; or 100-300- $\mu \mathrm{m}$ Embosphere, Merit Medical Manufacturing, Houston, Texas). The technique of PAE regarding the position of the microcatheter (distal vs proximal), baseline prostate volume, amount of embolic volume used, and the presence of pre-PAE acute urinary retention varied from patient to patient. Thus, it seems that the following question would be: Is smaller better? Actually, it has been shown in dogs that smaller $(100-300-\mu \mathrm{m})$ spherical embolic agents may have worse outcomes, with potential for urethral wall injury with peri-urethral frank necrosis and prostaticourethral fistula formation (6). PAE with $100-300-\mu \mathrm{m}$ spherical embolic agents failed to provide better outcomes than PAE with $300-500-\mu \mathrm{m}$ spherical embolic agents in a prospective RCT with 15 patients on each arm (7). Adverse events were more frequent with the smaller-sized embolics; however, the small sample size limited any significant conclusions. 
Larger prospective trials comparing spherical embolic sizes for PAE are awaited in later issues of this journal.

A word of caution has to be said when generalizing these size considerations for all types of embolic agents. Polyvinyl alcohol (PVA) particles have also been extensively used for PAE with similar results to spherical embolic agents (8). Smaller sizes of PVA did not induce more adverse events after PAE and may lead to better functional outcomes, with conflicting evidence regarding symptomatic relief $(9,10)$. It is likely that the tendency for clumping and upsizing "protect" the peri-urethral prostate from extensive necrosis with the smaller-sized PVA particles. There are no reports so far of CGD with PVA of any size or spherical embolics above $300 \mu \mathrm{m}$ in size.

CGD is due to more extensive necrosis of the periurethral central gland tissue and has the potential for longer symptomatic relief (yet not proven thus far) with greater prostate volume reduction, $\mathrm{Q}_{\max }$ increase, and PVR reduction, leading to a higher proportion of unobstructed patients after PAE (1). On the downside, it is associated with more pain in the week after PAE and may lead to urinary tract obstruction and/or infection needing endoscopic bailout $(1,3-5)$. Anejaculation or decrease in ejaculate volume is also a highly likely scenario with post-PAE CGD. When looking at these scenarios and the magnetic resonance images after CGD (1), it seems that PAE is getting more and more similar to TURP. Is this a good thing? It is not clear. If one wanted to have a technique that is the same as TURP, then the need for such a technique would not be obvious, as TURP has been present for decades and remains the gold standard for symptomatic relief in selected patients with $\mathrm{BPH}$. With more prostate tissue destruction comes more adverse events; this is inevitable. We probably want to envision PAE as a minimally invasive technique that is better than medical therapy. It may not be not as good at relieving symptoms and urinary obstruction as TURP, but it clearly has a lower adverse event profile. The low adverse event profile of PAE has recently been questioned, especially regarding ejaculatory dysfunction after embolization (11). Ejaculatory dysfunction after PAE was reported in $56 \%$ of patients in the Swiss RCT (2) and in $28 \%$ of the UKROPE trial participants (12). Little is actually known about these patients, as ejaculatory function data were not prospectively collected and were obtained through telephone calls in subgroups of patients without objective evaluation through validated questionnaires. Many of these patients were asked through telephone calls about their impression regarding ejaculation after PAE. Little detail was provided about the embolic agents used, preexisting ejaculatory dysfunction, and the medication of these patients both prior to and after PAE that could possibly influence ejaculatory status. Also, selection bias is possible as only part of the studied cohorts had ejaculatory evaluation. However, a prospective phase II trial assessing the safety and efficacy of PAE for symptomatic BPH in 45 patients looked specifically at erectile function and ejaculatory function and failed to demonstrate any negative outcomes after embolization (13).
Again, all patients were embolized with $300-500-\mu \mathrm{m}$ spherical embolic agents without any use of spherical embolic agents under $300 \mu \mathrm{m}$. Another prospective phase II trial of PAE in 51 patients with symptomatic BPH using $250-\mu \mathrm{m}$ spherical embolic agents reported 5 men $(9.8 \%)$ with dry or reduced ejaculate volume after PAE (14). Four of these patients had new onset after PAE but resolved within 6 months. The remaining patient already reported reduced ejaculate as a side effect of prostatic medication that did not resolve after PAE. The reasons for reduced ejaculate after PAE may be multiple, including retrograde ejaculation due to the destruction of the bladder neck sphincter; reduction of ejaculate volume due to the destruction of the prostatic glands with reduced prostate volume; nontarget embolization of seminal vesicles; and ischemic fibrosis of the ejaculatory ducts, among others. Histologic samples would be needed to assess this hypothesis.

What about even smaller embolic agents? Limited data are known about PAE using $100-\mu \mathrm{m}$ spherical embolic agents, but a recently published phase II trial of PAE for prostate cancer reported a $17 \%$ rate of bladder ischemia (2/12 patients) caused by embolization, requiring additional surgery for repair (15). The future of PAE among all medical and surgical therapeutic options for symptomatic BPH will probably reside within the low adverse event profile. As such, future trials on PAE should focus on the adverse event profile of the technique, with validated prospective collection of erectile and ejaculatory function and comparisons of different embolic agents and sizes with long-term follow-up. The potential for ejaculatory dysfunction after PAE with spherical embolic agents under $300 \mu \mathrm{m}$ and a higher adverse event profile with limited symptomatic gain questions its standard use, even if functional outcomes might be better. There is no clear benefit in providing an "endovascular resection of the prostate," as the traditional endoscopic route has proven to be very effective for the last decades.

\section{REFERENCES}

1. Hechelhammer L, Müllhaupt G, Mordasini L, et al. Predictability and inducibility of detachment of prostatic central gland tissue after prostatic artery embolization: post hoc analysis of a randomized controlled trial. J Vasc Interv Radiol 2019; 30:217-224.

2. Abt D, Hechelhammer L, Müllhaupt G, et al. Comparison of prostatic artery embolisation (PAE) versus transurethral resection of the prostate (TURP) for benign prostatic hyperplasia: randomised, open label, noninferiority trial. BMJ 2018; 361:k2338.

3. Costa NV, Pereira J, Fernandes L, Bilhim T, Pisco JM. Prostatic tissue expulsion after prostatic artery embolization. J Vasc Interv Radiol 2016; 27:601-603

4. Leite LC, de Assis AM, Moreira AM, Harward SH, Antunes AA, Carnevale FC. Prostatic tissue elimination after prostatic artery embolization (PAE): a report of three cases. Cardiovasc Intervent Radiol 2017; 40:937-941

5. Ghelfi J, Poncet D, Sengel C, et al. Prostatic fragment requiring endoscopic management after prostatic artery embolization for indwelling bladder catheter. Cardiovasc Intervent Radiol 2018; 41:1295-1297.

6. Brook OR, Faintuch S, Brook A, Goldberg SN, Rofsky NM, Lenkinski RE. Embolization therapy for benign prostatic hyperplasia: influence of embolization particle size on gland perfusion. J Magn Reson Imaging 2013; 38:380-387. 
7. Gonçalves OM, Carnevale FC, Moreira AM, Antunes AA, Rodrigues VC, Srougi M. Comparative study using 100-300 versus 300-500 $\mu \mathrm{m}$ microspheres for symptomatic patients due to enlarged-BPH prostates. Cardiovasc Intervent Radiol 2016; 39:1372-1378.

8. Bilhim T, Pisco J, Pereira JA, et al. Predictors of clinical outcome after prostate artery embolization with spherical and nonspherical polyvinyl alcohol particles in patients with benign prostatic hyperplasia. Radiology 2016; 281:289-300.

9. Bilhim T, Pisco J, Campos Pinheiro L, et al. Does polyvinyl alcohol particle size change the outcome of prostatic arterial embolization for benign prostatic hyperplasia? Results from a single-center randomized prospective study. J Vasc Interv Radiol 2013; 24:1595-1602.e1.

10. Wang MQ, Zhang JL, Xin HN, et al. Comparison of clinical outcomes of prostatic artery embolization with $50-\mu \mathrm{m}$ plus $100-\mu \mathrm{m}$ polyvinyl alcohol (PVA) particles versus 100- $\mu \mathrm{m}$ PVA particles alone: a prospective randomized trial. J Vasc Interv Radiol 2018; 29:1694-1702.

11. Madersbacher S. Re: Comparison of prostatic artery embolization (PAE) versus transurethral resection of the prostate (TURP) for benign prostatic hyperplasia: randomised, open label, non-inferiority trial. Eur Urol 2018; 74:844.

12. Ray AF, Powell J, Speakman MJ, et al. Efficacy and safety of prostate artery embolization for benign prostatic hyperplasia: an observational study and propensity-matched comparison with transurethral resection of the prostate (the UK-ROPE study). BJU Int 2018; 122:270-282.

13. Salem R, Hairston J, Hohlastos E, et al. Prostate artery embolization for lower urinary tract symptoms secondary to benign prostatic hyperplasia: results from a prospective FDA-approved investigational device exemption study. Urology 2018; 120:205-210.

14. Brown N, Walker D, McBean R, et al. Prostate artery Embolisation Assessment of Safety and feasibilitY (P-EASY): a potential alternative to long-term medical therapy for benign prostate hyperplasia. BJU Int 2018; 122(Suppl 5):27-34.

15. Mordasini L, Hechelhammer L, Diener PA, et al. Prostatic artery embolization in the treatment of localized prostate cancer: a bicentric prospective proof-of-concept study of 12 patients. J Vasc Interv Radiol 2018; 29: 589-597. 